\title{
Elliptic Flow of Thermal Photons in Relativistic Nuclear Collisions
}

\author{
Rupa Chatterjee, ${ }^{1}$ Evan S. Frodermann, ${ }^{2}$ Ulrich Heinz, ${ }^{2}$ and Dinesh K. Srivastava ${ }^{1}$ \\ ${ }^{1}$ Variable Energy Cyclotron Centre, 1/AF Bidhan Nagar, Kolkata 700 064, India \\ ${ }^{2}$ Physics Department, The Ohio State University, Columbus, Ohio 43210, USA
}

(Received 28 November 2005; published 26 May 2006)

\begin{abstract}
We predict the transverse momentum $\left(p_{T}\right)$ dependence of elliptic flow of thermal photons for $\mathrm{Au}+\mathrm{Au}$ collisions at the BNL Relativistic Heavy Ion Collider. We model the system hydrodynamically, with a thermalized quark-gluon plasma at early times followed by hadronization and decoupling. Photons are emitted throughout the expansion history. Contrary to hadron elliptic flow, which increases monotonically with $p_{T}$, the elliptic flow $v_{2}\left(p_{T}\right)$ of thermal photons is predicted to first rise and then fall again. Photon elliptic flow at high $p_{T}$ reflects the quark momentum anisotropy at early times when it is small, while at low $p_{T}$ it mirrors the large pion momentum anisotropy during the late hadronic emission stage. An interesting structure is predicted at intermediate $p_{T} \sim 0.4 \mathrm{GeV} / c$, where photon elliptic flow reflects the momenta and the (compared to pions) reduced $v_{2}$ of heavy vector mesons in the late hadronic phase.
\end{abstract}

DOI: 10.1103/PhysRevLett.96.202302

PACS numbers: $25.75 .-\mathrm{q}, 12.38 . \mathrm{Mh}$

Experiments performed at the Relativistic Heavy Ion Collider (RHIC) are providing evidence for the production of quark-gluon plasma (QGP) in nuclear collisions at ultrarelativistic energies. Key findings include strong anisotropic flow of all hadronic species [1-3] and a suppression of high- $p_{T}$ hadrons due to parton energy loss in the dense medium $[4,5]$. Signatures of direct photon emission [6-9], indicative of a hot early state, have also started emerging [10]. The emphasis of the next generation of experiments will thus necessarily shift to a more precise determination of the properties of both the QGP and the subsequent hot hadronic matter. Hydrodynamic flow, and, in particular, anisotropic flow in noncentral collisions, provides strong evidence for the existence of a hot and dense initial state with thermal pressure [3]. Elliptic flow is generated very early, via the transformation of the initial spatial eccentricity of the nuclear overlap region into momentum anisotropies, through the action of azimuthally anisotropic pressure gradients. With the passage of time, the pressure gradients equalize, and the growth of elliptic flow shuts itself off [11].

Because of their strong interactions, hadrons decouple from the system late, typically when the temperature has dropped to values around $100 \mathrm{MeV}$ [3,12]. In hydrodynamic simulations, hadrons with large transverse momenta are thus emitted from those fluid elements which have the largest radial flow. This is supported by the hydrodynamically predicted $p_{T}$ dependence of the elliptic flow coefficient $v_{2}$ and of the Hanbury Brown-Twiss radii [12]. Photons, on the other hand, are emitted at every stage of the collision, from the preequilibrium stage [8], the quarkgluon fluid, and the late hadronic matter. The thermal emission of photons from the QGP and hadronic phases is obtained [13] by integrating the thermal emission rate (which is strongly biased towards higher temperatures) over the space-time history of the system. As a result, high- $p_{T}$ photons arise mostly from the hot early stage, where hydrodynamic flow is weak but the spatial eccentricity of the source is large, whereas hadrons are emitted when the temperature is low, the flow is strong and anisotropic, but the spatial eccentricity of the fireball has mostly disappeared. The elliptic flow of photons, especially at $p_{T} \geq 1-2 \mathrm{GeV} / c$, is therefore expected to provide a glimpse of the early part of the expansion history when the fireball is in the QGP phase, complementary to the elliptic flow of hadrons.

This last argument can be made independent of the validity of hydrodynamics. For two of the leading photon production processes (quark-gluon Compton scattering and quark-antiquark annihilation for high energy photons in the QGP, $\pi \pi \rightarrow \rho \gamma$ for low energy photons in the hadronic phase) it is known that, for photon energies well above the rest masses of the emitting particle, the photon production cross section peaks very strongly for momenta close to that of the emitting particle [14]. So, even without local thermal equilibrium, one expects the photon elliptic flow to track the momentum anisotropy of the photonemitting particles at similar momenta.

For $\mathrm{Au}+\mathrm{Au}$ collisions at RHIC it is known $[2,15]$ that the hydrodynamic behavior of elliptic flow begins to break down for mesons (baryons) above $p_{T} \simeq 1.5 \mathrm{GeV} / c$ $(2.3 \mathrm{GeV} / c)$ : instead of continuing to rise with $p_{T}$, as predicted by hydrodynamics [3], the elliptic flow saturates. On the other hand, the systematics of hadron production with $p_{T} \gtrsim 2 \mathrm{GeV} / c$ can be well described by quark coalescence [16]. The observations then translate into a quark elliptic flow near hadronization that breaks away from hydrodynamics above $p_{T} \simeq 0.75 \mathrm{GeV} / c$ [15]. Thus, even though ideal fluid dynamics excellently describes the bulk of particle production at RHIC, viscous corrections become significant at transverse momenta above $1 \mathrm{GeV} / c$ for quarks and gluons and above $2-3 \mathrm{GeV} / c$ for hadrons. We expect these nonequilibrium features to be reflected by the photons, and the hydrodynamic prediction for photon 
elliptic flow presented here to be only an upper limit once $p_{T}$ exceeds $1 \mathrm{GeV} / c$. Nevertheless, the qualitative features pointed out below are generic and expected to be robust against nonequilibrium corrections.

The photon momentum spectrum can be written as

$$
E d N_{\gamma} / d^{3} p=\int[(\ldots) \exp (-p \cdot u(x) / T(x))] d^{4} x .
$$

The square brackets indicate the thermal emission rate from the QGP or hadronic matter. $p^{\mu}=\left(p_{T} \cosh Y\right.$, $\left.p_{T} \cos \phi, p_{T} \sin \phi, p_{T} \sinh Y\right)$ denotes the 4-momentum of the photon, while the 4-velocity of the flow field is $u^{\mu}=\gamma_{T}\left(\cosh \eta, v_{x}(x, y), v_{y}(x, y), \sinh \eta\right) \quad$ (with $\quad \gamma_{T}=$ $\left(1-v_{T}^{2}\right)^{-1 / 2}, \quad v_{T}^{2}=v_{x}^{2}+v_{y}^{2}$, assuming boost-invariant longitudinal expansion. We use coordinates $\tau, x, y, \eta$, with volume element $d^{4} x=\tau d \tau d x d y d \eta$, where $\tau=$ $\left(t^{2}-z^{2}\right)^{1 / 2}$ is the longitudinal proper time and $\eta=$ $\tanh ^{-1}(z / t)$ is the space-time rapidity. The photon momentum is parametrized by its rapidity $Y$, transverse momentum $p_{T}=\left(p_{x}^{2}+p_{y}^{2}\right)^{1 / 2}$, and azimuthal emission angle $\phi$. The photon energy in the local fluid rest frame, which enters as $p \cdot u / T$ into the Boltzmann and several other factors in the thermal emission rate, is given by

$$
\frac{p \cdot u}{T}=\frac{\gamma_{T} p_{T}}{T}\left[\cosh (Y-\eta)-v_{T} \cos \left(\phi-\phi_{v}\right)\right],
$$

where $\phi_{v}=\tan ^{-1}\left(v_{y} / v_{x}\right)$ is the azimuthal angle of the transverse flow vector. Equation (2) shows that the azimuthal anisotropy ( $\phi$ dependence) of the photon spectrum, conventionally characterized by its Fourier coefficients $v_{n}$ (where for equal nuclei only even $n$ contribute at $Y=0$ ),

$$
\frac{d N(b)}{d^{2} p_{T} d Y}=\frac{d N(b)}{2 \pi p_{T} d p_{T} d Y}\left[1+2 v_{2}\left(p_{T}, b\right) \cos (2 \phi)+\cdots\right]
$$

is controlled by an interplay between collective flow anisotropy and geometric deformation of the temperature field $T(x, y, \tau)$ : It obviously vanishes in the absence of radial flow, $v_{T}=0$, but for nonzero $v_{T}$ it can arise from an anisotropic flow field or from an azimuthally deformed temperature field. Only if the transverse flow points radially $\left[\phi_{v}=\phi_{r}=\tan ^{-1}(y / x)\right]$ and both the flow and temperature fields are azimuthally symmetric $\left[v_{T}(x, y)=v_{T}(r)\right.$ and $T(x, y)=T(r)$ with $\left.r^{2}=x^{2}+y^{2}\right]$ can the $\phi$ dependence in Eq. (2) be made to disappear, by rotating in (1) $\boldsymbol{r} \equiv(x, y)=\left(r, \phi_{r}\right)$ such that $\phi_{r}=\phi$.

We employ the boost-invariant hydrodynamic code AZHYDRO [17] which has been used extensively to explore hadron production at RHIC [12]. We use standard [12,17] initial conditions for $\mathrm{Au}+\mathrm{Au}$ collisions at $\sqrt{s}=$ $200 \mathrm{~A} \mathrm{GeV}$, but extrapolated from the usual initial time $\tau_{0}=0.6 \mathrm{fm} / c$ to a 3 times smaller value of $\tau_{0}=$ $0.2 \mathrm{fm} / c$, assuming one-dimensional boost-invariant expansion between these times. We do so in order to account for at least a fraction of the preequilibrium photon production at very early times [18]; its contribution to the photon spectrum is important at large $p_{T}$, and will suppress $v_{2}^{\text {photon }}$ there because very little transverse flow develops before $0.6 \mathrm{fm} / c$. So our initial maximum entropy density in the center of the fireball for $b=0$ collisions is $s_{0}=$ $3 \times 117 \mathrm{fm}^{-3}=351 \mathrm{fm}^{-3}$, corresponding to a peak initial temperature of $T_{0}=520 \mathrm{MeV}$. The initial transverse entropy density profile is computed from the standard Glauber model parametrization [12].

We assume creation of a thermally and chemically equilibrated plasma at $\tau_{0}$, and use the complete leadingorder rate for QGP photon production from Ref. [6] and the latest results for photon radiation by a hot hadron gas from Ref. [7]. These rates provide good descriptions of singlephoton data at SPS energies and recently at RHIC $[9,19,20]$. We use the equation of state $\operatorname{EOS} Q$ [17] which matches a noninteracting QGP to a chemically equilibrated hadron gas at $T_{c}=164 \mathrm{MeV}$, with energy densities $\epsilon_{\mathrm{q}}\left(T_{c}\right)=1.6 \mathrm{GeV} / \mathrm{fm}^{3}$ and $\epsilon_{\mathrm{h}}\left(T_{c}\right)=0.45 \mathrm{GeV} / \mathrm{fm}^{3}$ in the subphases. Hadron freezeout is assumed to happen at $\epsilon_{\mathrm{f}}=0.075 \mathrm{GeV} / \mathrm{fm}^{3}$ [12].

Figure 1 shows the changing spatial anisotropy of the fireball, for $\mathrm{Au}+\mathrm{Au}$ collisions at a typical impact parameter of $b=7 \mathrm{fm}$, by plotting cuts through constant energy density surfaces along the $x$ and $y$ axes (i.e., in the reaction plane and perpendicular to it) for the three values $\epsilon_{\mathrm{q}}, \epsilon_{\mathrm{h}}$, and $\epsilon_{\mathrm{f}}$. After about $9 \mathrm{fm} / c$ the initial out-of-plane deformation changes sign (dashed and solid lines cross), as a result of faster expansion into the reaction plane. Figure 2 shows the flow velocities along the same cuts, illustrating the developing anisotropy of the transverse flow field. Through most of the fireball interior the velocity along the $x$ axis is larger and rises more rapidly with increasing radius than along the $y$ axis. At hadronic freezeout, $\epsilon_{\mathrm{f}}$, the in-plane and out-of-plane velocity gradients have almost (but not fully) equalized, and larger in-plane flow velocities exist mostly near the fireball surface. Figure 2 complements similar plots in Refs. [3,12] which show velocity

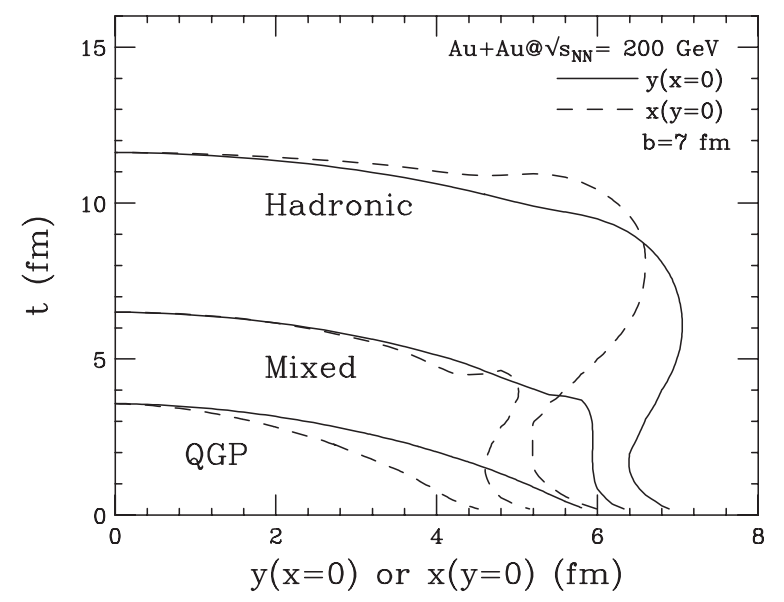

FIG. 1. Constant energy density contours for $\epsilon=\epsilon_{\mathrm{q}}, \epsilon_{\mathrm{h}}$, and $\epsilon_{\mathrm{f}}$, along $y(x=0)$ and $x(y=0)$, for $\mathrm{Au}+\mathrm{Au}$ at $b=7 \mathrm{fm}$. 


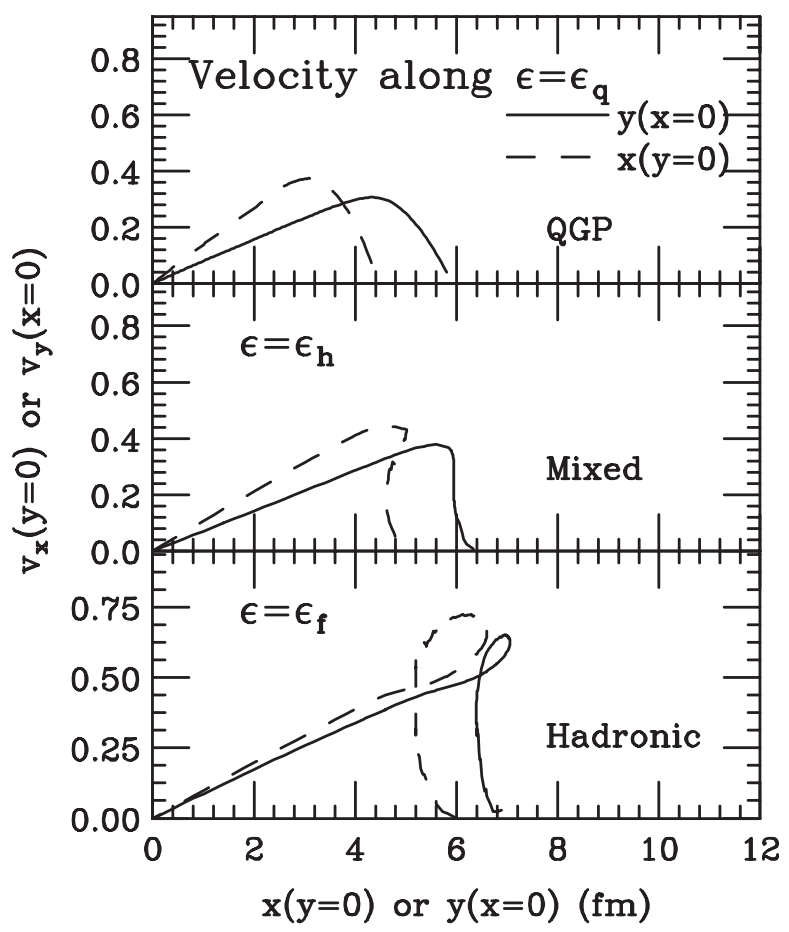

FIG. 2. Flow velocity along the constant energy density contours for $\epsilon=\epsilon_{\mathrm{q}}$ (QGP phase, upper panel), $\epsilon_{\mathrm{h}}$ (mixed phase, middle panel), and $\epsilon_{\mathrm{f}}$ (hadronic phase, lower panel) for $y(x=0)$ and $x(y=0)$, for $200 A \mathrm{GeV} \mathrm{Au}+\mathrm{Au}$ at $b=7 \mathrm{fm}$.

profiles either at constant time or only along the freezeout surface at $b=0$.

In Fig. 3 we show the thermal photon elliptic flow for $\mathrm{Au}+\mathrm{Au}$ collisions at $b=7 \mathrm{fm}$. We compare the overall $v_{2}\left(p_{T}\right)$ with the individual elliptic flow coefficients associated with quark matter $\left[v_{2}(\mathrm{QM})\right]$ and hadronic matter $\left[v_{2}(\mathrm{HM})\right]$ contributions to the thermal $\gamma$ spectrum. Comparison of $v_{2}(\mathrm{HM})$ with the elliptic flow of thermal $\pi$ and $\rho$ mesons, $v_{2}(\pi)$ and $v_{2}(\rho)$, shows that at low (high)

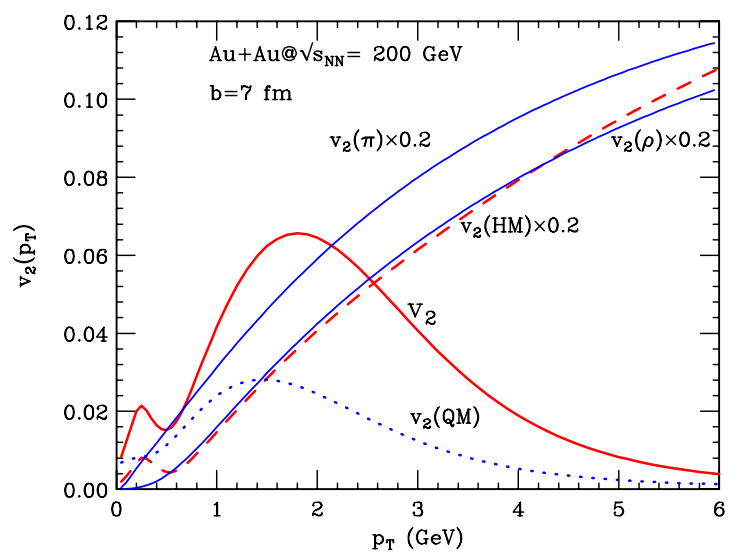

FIG. 3 (color online). $\quad v_{2}\left(p_{T}\right)$ for thermal photons from offcentral $200 A \mathrm{GeV} \mathrm{Au}+\mathrm{Au}$ collisions at $b=7 \mathrm{fm}$. Quark and hadronic matter contributions are also shown separately, and the elliptic flow of $\pi$ and $\rho$ mesons is shown for comparison.
$p_{T}$ hadronic photon flow tracks the elliptic flow of $\pi(\rho)$ mesons. This can be understood from the single-photon spectrum (see, e.g., Fig. 17 in [21]): at low $p_{T}$ hadronic photon emission is dominated by the processes $\rho \rightarrow \pi \pi \gamma$ and $\pi \pi \rightarrow \rho \gamma$ whereas at higher $p_{T}$ collision-induced conversion of vector mesons into photons (such as $\pi \rho \rightarrow$ $\pi \gamma$ ) takes over. In the present situation, with radial flow effects on the spectra computed from hydrodynamics, this transition happens around $p_{T} \sim 0.4 \mathrm{GeV} / c$. At $p_{T} \gtrsim$ $0.4 \mathrm{GeV} / c$ hadronic photon elliptic flow thus tracks the $v_{2}$ of $\rho$ and other vector mesons. Since the heavier vector mesons carry less elliptic flow than the lighter pions [3], this transition manifests itself in a reduction of hadronic photon $v_{2}$ around $p_{T} \sim 0.4 \mathrm{GeV} / c$ (see dashed red line in Fig. 3). Because of the dominance of hadronic processes in the total photon yield in this $p_{T}$ region, this structure survives in the total photon elliptic flow (solid red line).

The elliptic flow of the QGP photons, $v_{2}(\mathrm{QM})$, is small at low $p_{T}$, peaks around $p_{T} \sim 1.5 \mathrm{GeV} / c$, and decreases again for large $p_{T}$. Its smallness at high $p_{T}$ and increase towards lower $p_{T}$ reflects the absence of transverse flow during the earliest, hottest stage of the QGP and its gradual buildup during the following cooler stages. That growth is cut off by the generic decrease of $v_{2}$ as $p_{T} \rightarrow 0$ [22]. Its nonzero limit at $p_{T}=0$ (first observed in [23] for gluons) can be traced to the singularities of the Bose distribution and emission rates at $p_{T}=0$. Since the QGP photon rates [6] are unreliable for $p_{T}<0.2 \mathrm{GeV} / c$, the $v_{2}(\mathrm{HM})$ curve in Fig. 3 should not be trusted in that domain. Fortunately, there the total $v_{2}$ (solid red line) is entirely dominated by the hadronic photon contribution.

Although the hydrodynamically predicted elliptic flow of hadronic photons is almost everywhere much larger than that of the QGP photons, the hadronic contribution to the photon spectrum is increasingly suppressed (by more than an order of magnitude below the QGP contribution) once $p_{T}$ exceeds $1.5-2 \mathrm{GeV} / c$. Hence the overall photonic $v_{2}$, while larger than the pure QGP contribution, decreases for large $p_{T}$, too, approaching the $v_{2}$ of the QGP photons. At high $p_{T}$ the photon elliptic flow thus opens a window onto the dynamics of the QGP, in spite of the larger elliptic flow of the hadronic photons.

In Fig. 4 we present the impact parameter dependence of the elliptic flow of thermal photons. The impact parameters are chosen to roughly correspond to collision centralities of $0 \%-10 \%, 10 \%-20 \%, 20 \%-30 \%, 30 \%-40 \%, 40 \%-50 \%$, and $50 \%-60 \%$ of the total inelastic nuclear cross section. As the impact parameter increases, the relative contribution of hadronic photons increases, too. Since its elliptic flow is larger than that of the QGP photons, the $b$ dependence of thermal photon $v_{2}$ is predicted to be stronger than that of hadron $v_{2}$. This can be used as an additional tool to isolate the QGP contribution to thermal photon emission, especially if methods can be developed [24] to subtract at high $p_{T}$ the hadronic contribution $v_{2}(\mathrm{HM})$ from the ther- 


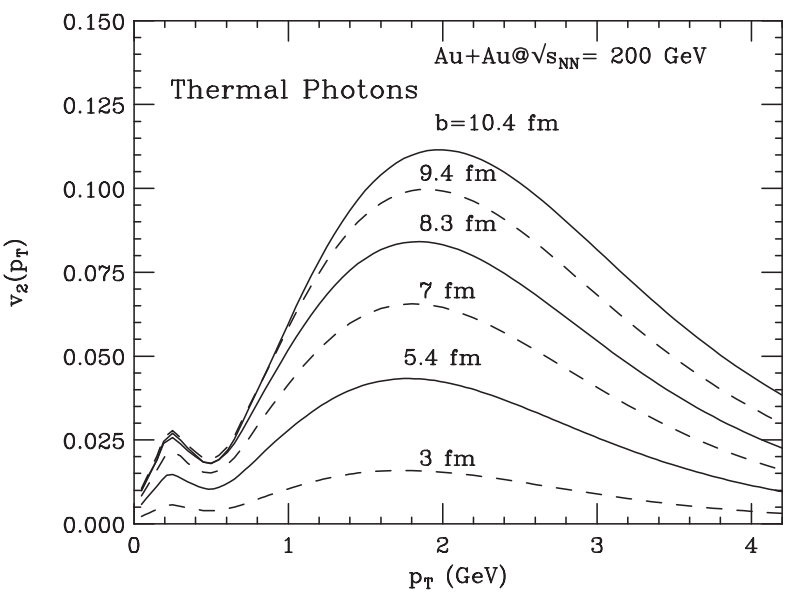

FIG. 4. Impact parameter dependence of the azimuthal anisotropy of thermal photons.

mal photon $v_{2}$, by exploiting its similarity to the elliptic flow of thermal $\rho$ mesons.

However, a quantitative interpretation of photon elliptic flow in the context of extracting dynamical information on the QGP evolution must also account for other contributions to high- $p_{T}$ photon production. It has recently been argued that jets passing through the QGP contribute significantly to direct photon production at large $p_{T}$ [9] and may even lead to negative photon elliptic flow in this region [25]. It will be interesting to see how much of this effect survives once the so far neglected anisotropic collective flow of the medium is included.

In conclusion, we have presented a first calculation of elliptic flow of thermal photons from ultrarelativistic heavy-ion collisions. The differential photon elliptic flow $v_{2}\left(p_{T}\right)$ exhibits a rich structure, driven by the evolution of the system and the competing rates of photon emission from the quark and hadronic matter stages. Its impact parameter dependence differs from that of pion elliptic flow since the relative contributions of hadronic and quark matter emissions change with collision centrality. At high $p_{T}$, where ideal fluid dynamics is known to overpredict $\boldsymbol{v}_{2}$, the elliptic flow of thermal photons from the hadronic matter tracks the flow of $\rho$ mesons which can be measured independently. If this can be used to subtract the hadronic contribution to photon elliptic flow at high $p_{T}$, a new window onto the early buildup of collective flow in the QGP will be opened.

This work was supported by the U.S. Department of Energy under Contract No. DE-FG02-01ER41190.

[1] C. Adler et al. (STAR Collaboration), Phys. Rev. Lett. 87, 182301 (2001); 89, 132301 (2002); J. Adams et al. (STAR Collaboration), ibid. 92, 052302 (2004); K. Adcox et al. (PHENIX Collaboration), ibid. 89, 212301 (2002).
[2] C. Adler et al. (STAR Collaboration), Phys. Rev. Lett. 90, 032301 (2003); S. S. Adler et al. (PHENIX Collaboration), ibid. 91, 182301 (2003).

[3] P. F. Kolb, J. Sollfrank, and U. Heinz, Phys. Rev. C 62, 054909 (2000); P. Huovinen, P. F. Kolb, U. Heinz, P. V. Ruuskanen, and S.A. Voloshin, Phys. Lett. B 503, 58 (2001); D. Teaney, J. Lauret, and E. V. Shuryak, nucl-th/ 0110037.

[4] X. N. Wang, Phys. Rev. C 63, 054902 (2001); M. Gyulassy, I. Vitev, and X. N. Wang, Phys. Rev. Lett. 86, 2537 (2001).

[5] K. Adcox et al. (PHENIX Collaboration), Phys. Rev. Lett. 88, 022301 (2002); J. Adams et al. (STAR Collaboration), ibid. 91, 172302 (2003).

[6] P. Arnold, G. D. Moore, and L. G. Yaffe, J. High Energy Phys. 12 (2001) 009.

[7] S. Turbide, R. Rapp, and C. Gale, Phys. Rev. C 69, 014903 (2004).

[8] S. A. Bass, B. Müller, and D. K. Srivastava, Phys. Rev. Lett. 90, 082301 (2003); Phys. Rev. C 66, 061902(R) (2002); T. Renk, S. A. Bass, and D. K. Srivastava, Phys. Lett. B 632, 632 (2006).

[9] R. J. Fries, B. Müller, and D. K. Srivastava, Phys. Rev. Lett. 90, 132301 (2003); Phys. Rev. C 72, 041902(R) (2005).

[10] S. S. Adler et al. (PHENIX Collaboration), Phys. Rev. Lett. 94, 232301 (2005).

[11] H. Sorge, Phys. Rev. Lett. 78, 2309 (1997).

[12] P. F. Kolb and U. Heinz, in Quark-Gluon Plasma 3, edited by R.C. Hwa and X.-N. Wang (World Scientific, Singapore, 2004), p. 634.

[13] Jan-e Alam, D. K. Srivastava, B. Sinha, and D. N. Basu, Phys. Rev. D 48, 1117 (1993).

[14] C. Y. Wong, Introduction to High-Energy Heavy-Ion Collisions (World Scientific, Singapore, 1994), p. 382.

[15] U. Heinz, AIP Conf. Proc. 739, 163 (2004).

[16] V. Greco, C. M. Ko, and P. Levai, Phys. Rev. Lett. 90, 202302 (2003); R. J. Fries, B. Müller, C. Nonaka, and S. A. Bass, ibid. 90, 202303 (2003); D. Molnar and S. A. Voloshin, ibid. 91, 092301 (2003).

[17] The code can be downloaded from URL http://nt3.phys. columbia.edu/people/molnard/OSCAR/. See also the first paper in [3], and P. F. Kolb and R. Rapp, Phys. Rev. C 67, 044903 (2003).

[18] S. A. Bass, B. Müller, and D. K. Srivastava, Phys. Rev. Lett. 93, 162301 (2004).

[19] D. K. Srivastava, Phys. Rev. C 71, 034905 (2005).

[20] D. d'Enterria and D. Peressounko, Eur. Phys. J. C 46, 451 (2006).

[21] C. Gale and K.L. Haglin, in Quark-Gluon Plasma 3, edited by R. C. Hwa and X.-N. Wang (World Scientific, Singapore, 2004), p. 364.

[22] P. Danielewicz, Phys. Rev. C 51, 716 (1995).

[23] U.Heinz and S.M.H. Wong, Phys. Rev. C 66, 014907 (2002).

[24] S. Adler et al. (PHENIX Collaboration), Phys. Rev. Lett. 96, 032302 (2006).

[25] S. Turbide, C. Gale, and R. J. Fries, Phys. Rev. Lett. 96, 032303 (2006). 